\title{
Final report SI 08-SI-004: Fusion application targets
}

J. Biener, S. O. Kucheyev, M. Y. Wang, C. Dawedeit, M. A. Worsley, S. H. Kim, C. Walton, G. Gilmer, L. Zepeda-Ruiz, A. A. Chernov, J. I. Lee, T. M. Willey, M. M. Biener, T. van Buuren, K. J. Wu, J. H. Satcher, A. V. Hamza

December 6, 2010 
This document was prepared as an account of work sponsored by an agency of the United States government. Neither the United States government nor Lawrence Livermore National Security, LLC, nor any of their employees makes any warranty, expressed or implied, or assumes any legal liability or responsibility for the accuracy, completeness, or usefulness of any information, apparatus, product, or process disclosed, or represents that its use would not infringe privately owned rights. Reference herein to any specific commercial product, process, or service by trade name, trademark, manufacturer, or otherwise does not necessarily constitute or imply its endorsement, recommendation, or favoring by the United States government or Lawrence Livermore National Security, LLC. The views and opinions of authors expressed herein do not necessarily state or reflect those of the United States government or Lawrence Livermore National Security, LLC, and shall not be used for advertising or product endorsement purposes.

This work performed under the auspices of the U.S. Department of Energy by Lawrence Livermore National Laboratory under Contract DE-AC52-07NA27344. 


\section{Fusion Application Targets}

J. Biener, C. Dawedeit, A. A. Chernov, M. A. Worsley, S. H. Kim, J. I. Lee, T. M. Willey, S. O. Kucheyev, M. Y. Wang, M. M. Biener, T. van Buuren., K. J. Wu, J. H.

Satcher, Jr. and A. V. Hamza,

Nanoscale Synthesis and Characterization Laboratory,

Lawrence Livermore National Laboratory

7000 East Avenue, Livermore, CA 94550, USA

Biener2@llnl.gov

Complex target structures are necessary to take full advantage of the unique laboratory environment created by inertial confinement fusion experiments. For example, uses-ofignition targets that contain a thin layer of a low density nanoporous material inside a spherical ablator shell allow placing dopants in direct contact with the DT fuel. The ideal foam for this application is a low-density hydrocarbon foam that is strong enough to survive wetting with cryogenic hydrogen, and low enough in density (density less than $\sim 30 \mathrm{mg} / \mathrm{cc}$ ) to not reduce the yield of the target. Here, we discuss the fabrication foamlined uses-of-ignition targets, and the development of low-density foams that can be used for this application.

\subsubsection{Introduction}

Much effort has been directed over the last 20 years toward the development of spherical foam targets for direct-drive ${ }^{1-7}$ and fast-ignition ${ }^{8-10}$ experiments. In these targets, the spherical foam shell is used to define the shape of the cryogenic DT fuel layer, ${ }^{11}$ or acts as a surrogate to simulate the cryogenic fuel layer. These targets are fabricated from relatively high-density aerogels (>100 mg/cc) and coated with a few micron thick permeation barrier. With exception of the above mentioned fast ignition targets, ${ }^{8-10}$ the wall of these targets is typically larger than 100 microns. In contrast, the fusion application targets for indirect-drive experiments on NIF will require a much thinner foam shell surrounded by a much thicker ablator shell. The design requirements for both types of targets are compared in Table 1.

\begin{tabular}{|l|c|c|}
\hline \multicolumn{1}{|c|}{ Requirements } & $\begin{array}{c}\text { Direct drive } \\
\text { targets for omega }\end{array}$ & $\begin{array}{c}\text { Indirect drive fusion } \\
\text { application targets } \\
\text { for NIF }\end{array}$ \\
\hline Shell diameter [mm] & $\sim 0.9$ & 2 \\
\hline Thickness of the foam shell [micron] & $100-200$ & 15 \\
\hline Foam density [mg/cc] & $100-250$ & 30 \\
\hline Foam composition & RF & Ideally pure CH \\
\hline Permeation barrier/ablator thickness [micron] & $3-4$ & $100-150$ \\
\hline Permeation barrier/ablator material & $\begin{array}{c}\text { Glow discharge } \\
\text { polymer (GDP) }\end{array}$ & $\begin{array}{c}\text { GDP, Be, High-density } \\
\text { Carbon (HDC) }\end{array}$ \\
\hline
\end{tabular}

Table1: Comparison of ICF targets for direct drive experiments on OMEGA and NIF. 
The foam shell targets for direct-drive experiments can be made in large quantities and with reasonably high yields using an encapsulation technique pioneered by Takagi et al. in the early 90 's. ${ }^{4}$ In this approach, targets are made by first generating unsupported foam shells using a triple-orifice droplet generator, followed by coating the dried foam shells with a thin permeation barrier. However, this approach is difficult, if not impossible, to transfer to the lower density and thinner wall foam shells required for indirect-drive usesof-ignition targets for NIF that then would have to be coated with an at least hundredmicron-thick ablator film. So far, the thinnest shells that have been fabricated using the triple-orifice-droplet generator technique had a wall thickness of 20 microns, but despite of being made from a higher-density foam formulation, the shells were mechanically very sensitive, difficult to dry, and showed large deviations from roundness.

We thus decided to explore a different approach based on using prefabricated thickwalled spherical ablator shells as templates for the thin-walled foam shell. As in the case of the above mentioned encapsulation technique, the foam is made by sol-gel chemistry. However, our approach removes much the requirements on the mechanical stability of the foam shell as the foam shell is never handled in its free-standing form, and promises superior ablator uniformity and surface roughness. As discussed below, the success of this approach depends strongly on the availability of suitable aerogel chemistries (ideally pure hydrocarbon (CH)-based systems) with suitable rheological properties (high viscosity and high modulus near the gel point) that produce low-density and mechanically strong foams.

\section{Results and Discussions}

Our approach to fabricate foam-lined fusion application targets is schematically illustrated in Figure 1. The process involves the following steps: 1) filling of the ablator shell with the desired amount of the aerogel precursor solution, 2) temporarily plugging the fill hole, 3) film formation by randomly rotating the capsule during polymerization, and 4) solvent exchange and supercritical drying. In the following, we will focus mainly on the development of suitable aerogel chemistries that are compatible with the target specification listed in Table 1 and those rheological properties allow for film formation in a rotating capsule.

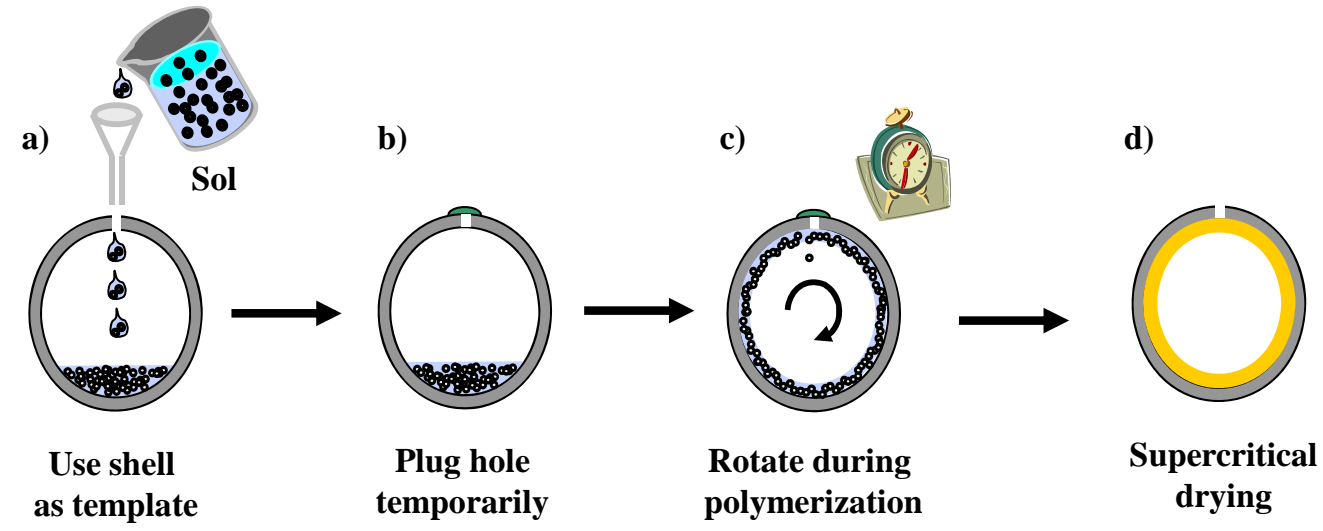

Figure 1: Chemistry-in-a-capsule approach proposed to fabricate foam-lined fusion application targets for the National Ignition 


\subsection{Dynamics of fluids in a rotating cylinder}

To explore the effect of film thickness and viscosity on film uniformity and shear, we studied the behavior of fluids in a rotating horizontal circular cylinder both theoretically and experimentally.

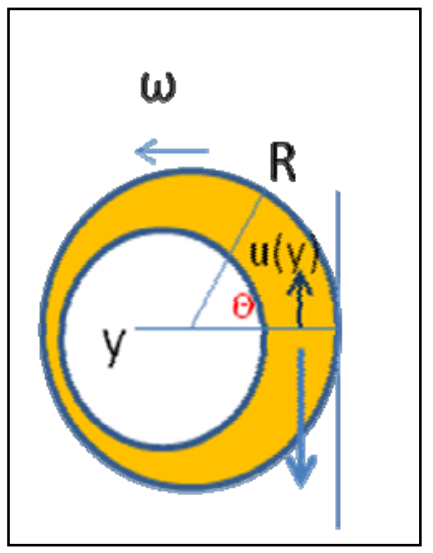

Figure 2: Illustration of the dynamics of a fluid in a rotating horizontal cylinder with a radius $R$ and an angular speed $\omega$. A continuous film can only be formed if the gravity induced down flow is smaller than the drag exhibited by the cylinder. The transition towards a smooth film is observed at the critical rotational velocity $\omega_{\text {crit }}$ $\omega_{\text {crit }} \sim g h^{2} / R v$,

where $g$ is the gravitational constant, $981 \mathrm{~cm} / \mathrm{s}^{2}, h$ is the average film thickness, and $v$ is the kinematic viscosity.

Neglecting surface tension and wetting effects, the problem of a fluid in a rotating horizontal cylinder can be solved analytically (Figure 2). The analysis reveals that 1 ) the critical rotational velocity $\omega_{\text {crit }}$ scales with the square of the film thickness $h$, and the inverse of both cylinder radius $R$ and viscosity $v, 2$ ) the film thickness inhomogenity $\Delta h$ scales as the third power of $h$, and the inverse of $R, v$ and angular speed $\omega$; and 3) the shear rate scales as the inverse of $v$. Assuming a radius of $1 \mathrm{~mm}$, a film thickness of 30 micron, and a viscosity of $\sim 1 \mathrm{cP}$ (water), the analytical solution of the problem predicts a critical rotational velocity of $200 \mathrm{rpm}$. If the viscosity at the gel point increases to $10^{3} \mathrm{cP}$ (as shown for the RF system ${ }^{12}$ ), then $\omega_{\text {crit }}$ decreases to less than $1 \mathrm{rpm}$, and the shear decreases by a factor of $10^{3}$. This analysis reveals the critical importance of controlling the viscosity of the precursor solution near the gel point for the quality of the film.
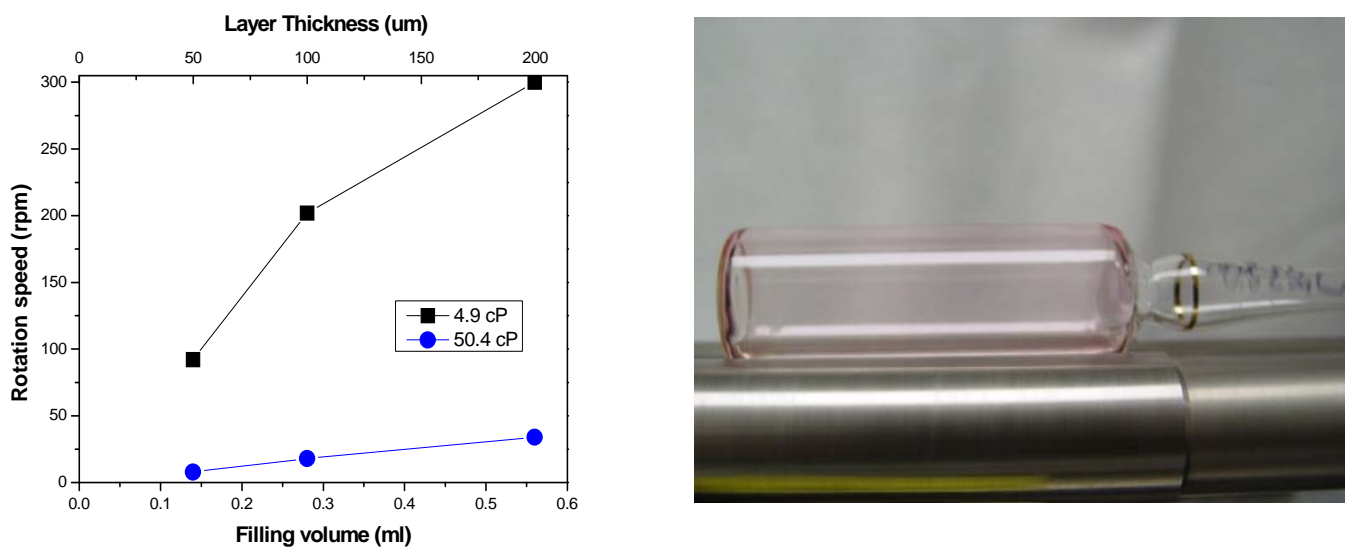

Figure3: (Left) Effect of viscosity and film thickness on the critical rotation speed required to form a uniform layer in a rotating horizontal circular cylinder. (Right) Example of a 200-micron-thick oil film (viscosity $4.9 \mathrm{cP}$ ) formed at $200 \mathrm{rpm}$. 
The experimental results are qualitatively consistent with the predictions of the analytical model: the critical rotation velocity decreases with increasing viscosity and decreasing film thickness, although the film thickness dependence is weaker than the expected square dependence (Figure 3).

\subsection{Aerogel systems}

\subsubsection{Resorcinal-formaldehyde polymer aerogels}

Most work on the encapsulation technique was preformed with the resorcinolformaldehyde (RF) system that was originally developed by Pekala et $a l .{ }^{13}$ at LLNL. Much progress has been made in understanding and controlling gel time, ${ }^{14}$ viscosity $^{2}$ and pore size ${ }^{14}$ of this polymer system. One of the reasons for selecting this material is its optical transparency that is a consequence of its small pore size $(<100 \mathrm{~nm})$ and allows for optical inspection of the cryogenic fuel layer. The major drawback of the RF chemistry for the ICF application is its high oxygen content $(\mathrm{O} / \mathrm{C}$ ratio $\sim 1 / 3)$ that is expected to reduce the yield of ICF targets. Using the triple orifice droplet generator technology, RF foam shell fabrication has been demonstrated for higher density $(<100$ $\mathrm{mg} / \mathrm{cc}$ ) and thicker shells (wall thickness $>100$ micron). Although 20-micron-thick shells have been produced for fast-ignition experiments, they are too dense (>130 mg/cc), mechanically very unstable and thus difficult to dry, and showed large deviations from roundness. ${ }^{8,10}$

To explore the spin-coating behavior of RF gels that is essential to our approach, we performed gelation tests in a rotating cylinder geometry. The observed formation of uniform and transparent gel layers (Figure 4) seems to be a consequence of the 1000-fold increase of the viscosity near the gel point of the RF solution that keeps the shear forces experienced by the gelling film low. The increase in viscosity of the RF system has been attributed to particle formation ${ }^{15}$

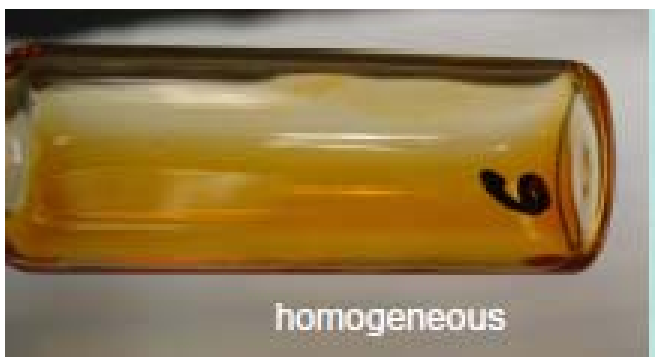

Figure 4: Example of an approximately 700 micron-tick RF gel film formed by slowly rotating cylinder filled with a high-viscosity, pre-polymerized RF solution( 22h@60 $\left.{ }^{\circ} \mathrm{C}\right)$.

\subsubsection{Hydrocarbon aerogels}

Driven by the desire to improve target performance, several pure $\mathrm{CH}$ foam systems have been developed for the foam shell application, including divinylbenzene (DVB) ${ }^{16}$ and poly(4-methyl-1-pentene) (PMP) foams. ${ }^{17}$ The densities, however, were considerably higher than required for the current application. We thus decided to develop a new hydrocarbon-based aerogel chemistry utilizing the ring opening metathesis polymerization (ROMP) to polymerize dicyclopentadiene (DCPD) monomers (Figure 5). ${ }^{18}$ As catalyst we selected the recently developed ruthenium-based Grubbs' catalyst ${ }^{19}$ that shows high metathesis activity and reasonable tolerance against air and moisture, and 
as solvent we use toluene. The resulting aerogels are monolithic, have densities as low as $30 \mathrm{mg} / \mathrm{cc}$, and are mechanically strong enough to survive wetting with cryogenic hydrogen as shown in Figure 6.

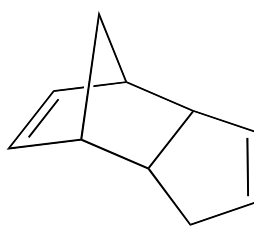

DCPD

monomer

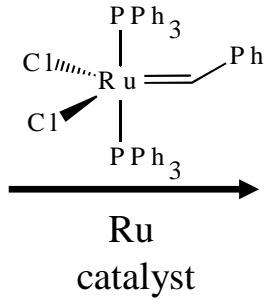

catalyst

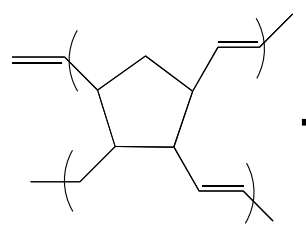

crosslinked polymer network

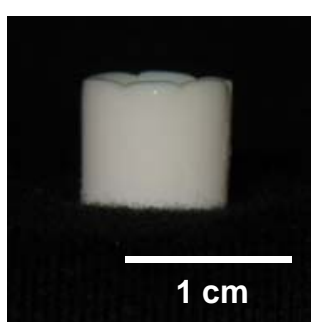

DCPD aerogel

Figure 5 Ring opening metathesis polymerization of dicyclopentadiene (DCPD) monomers with Grubbs’ catalyst. A $200 \mathrm{mg} / \mathrm{cc}$ DCPD aerogel is shown on the right.

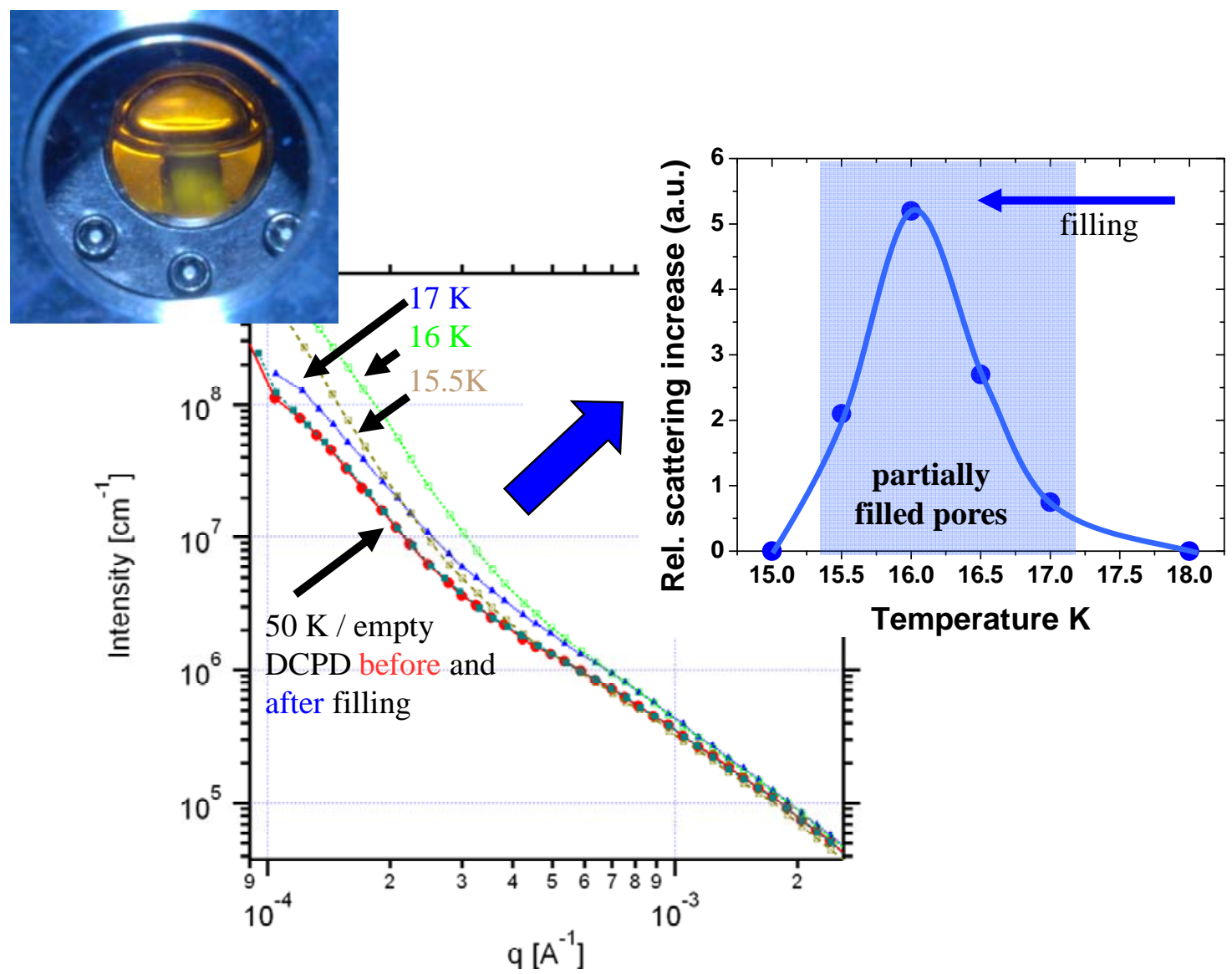

Figure 6: Wetting behavior of a DCPD aerogel (30 mg/cc) with cryogenic hydrogen. (Top) The DCPD sample stays monolithic upon wetting with liquid hydrogen. (Bottom) Small angle x-ray scattering (SAXS) data reveal the filling of the pore volume with cryogenic hydrogen. The scattering curves obtained from empty and hydrogen-filled DCPD are identical thus demonstrating the stability of the structure. 
We have fabricated targets that were completely filled with DCPD aerogel as well as partially filled targets that had a thin foam liner on the inside of the capsule. The formation of thin DCPD gel films in rotating capsules was not very robust and often resulted in inhomogeneous coatings. To address this issue we performed coating experiments in the rotating cylinder geometry. These experiments confirmed the formation of rough inhomogeneous films with a granular structure under rotation. This is contrast to the RF system where the formation of smooth and homogeneous gel films was observed under similar conditions. This observation suggests that the mechanical strength and/or viscosity of the DCPD system near to the sol-gel transition is too low to survive the shear forces that are a consequence of the rotating vial geometry. To proof this hypothesis we studied the rheological behavior of the DCPD system during gelation (Figure 7). These experiments show that the viscosity of the DCPD system does not increase during the initial stage of gelation, in contrast to the RF system that shows a 1000 -fold increase in viscosity before gelation starts.

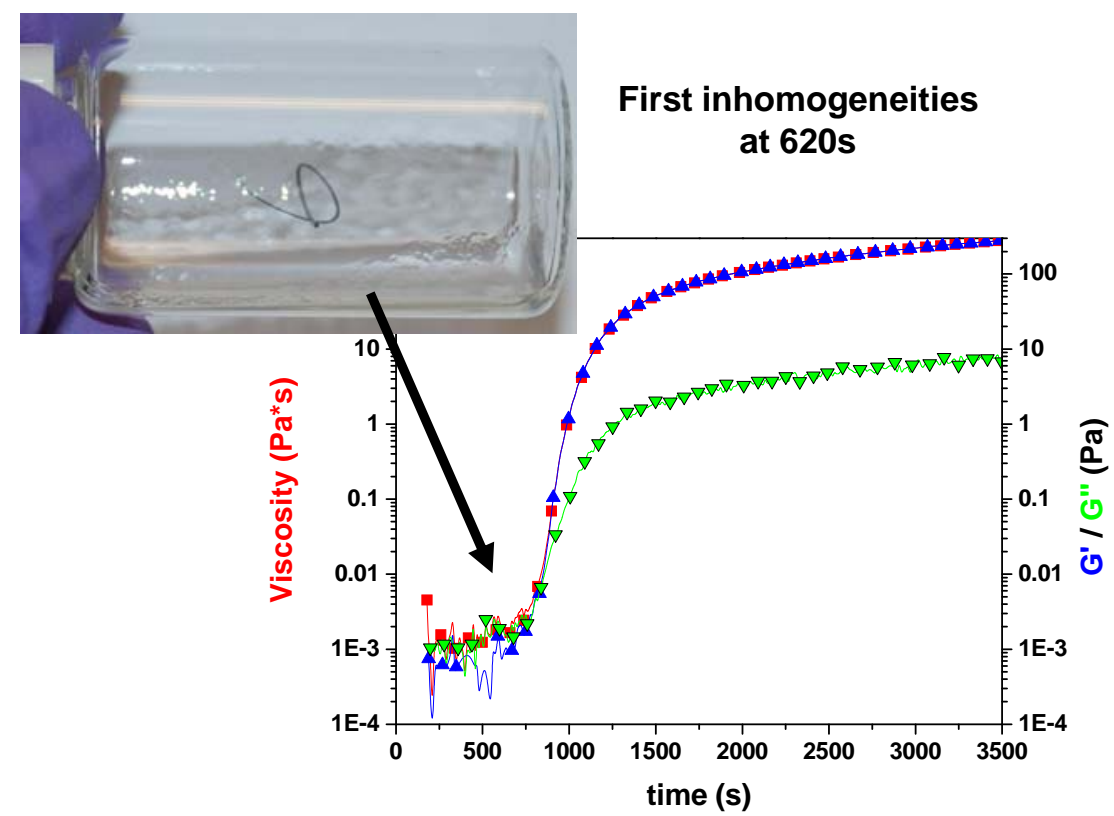

Figure 7: Rheological behavior during ROMP of DCPD ( 30 mg/cc). First inhomogenieties are observed before the viscosity increases thus indicating that the evolving DCPD network is too weak and fails under shear.

One possible explanation of the different rheological behavior of DCPD and RF is the different functionality of both systems, and thus the different polymerization behavior that comes with it. RF has a functionality of 3, and thus immediately forms a threedimensional cross-linked network, whereas DCPD has a functionality of two thus forming predominately linear chains (the reactivity of the second double bond in DCPD is lower due to its lower ring strain ${ }^{20}$ ). To force the DCPD system into a 3D cross-linked network morphology, and thus to increase both viscosity and strength at the sol-gel transition, we tested the addition of appropriate cross-linkers. ${ }^{21}$ Preliminary results were very promising, and showed the formation of homogeneous, transparent thins gel films in the rotating cylinder test geometry. An alternative explanation may be the limited 
solubility of the DCPD polymer network in toluene. If this is the case, one should be able to improve the stability of the DCPD system by increasing its solubility. Finally, the viscosity of the aerogel precursor solution can be independently controlled by using a higher viscosity solvent, and preliminary experiments using a paraffin-based solvent look promising.

\subsection{Layer formation and Random Positioning Machine}

The formation of smooth homogenous films inside a spherical capsule requires precise control over rotation speed and direction. Initial film formation experiments that used the tumbling motion of a capsule in a rotating vial were promising, but not robust.

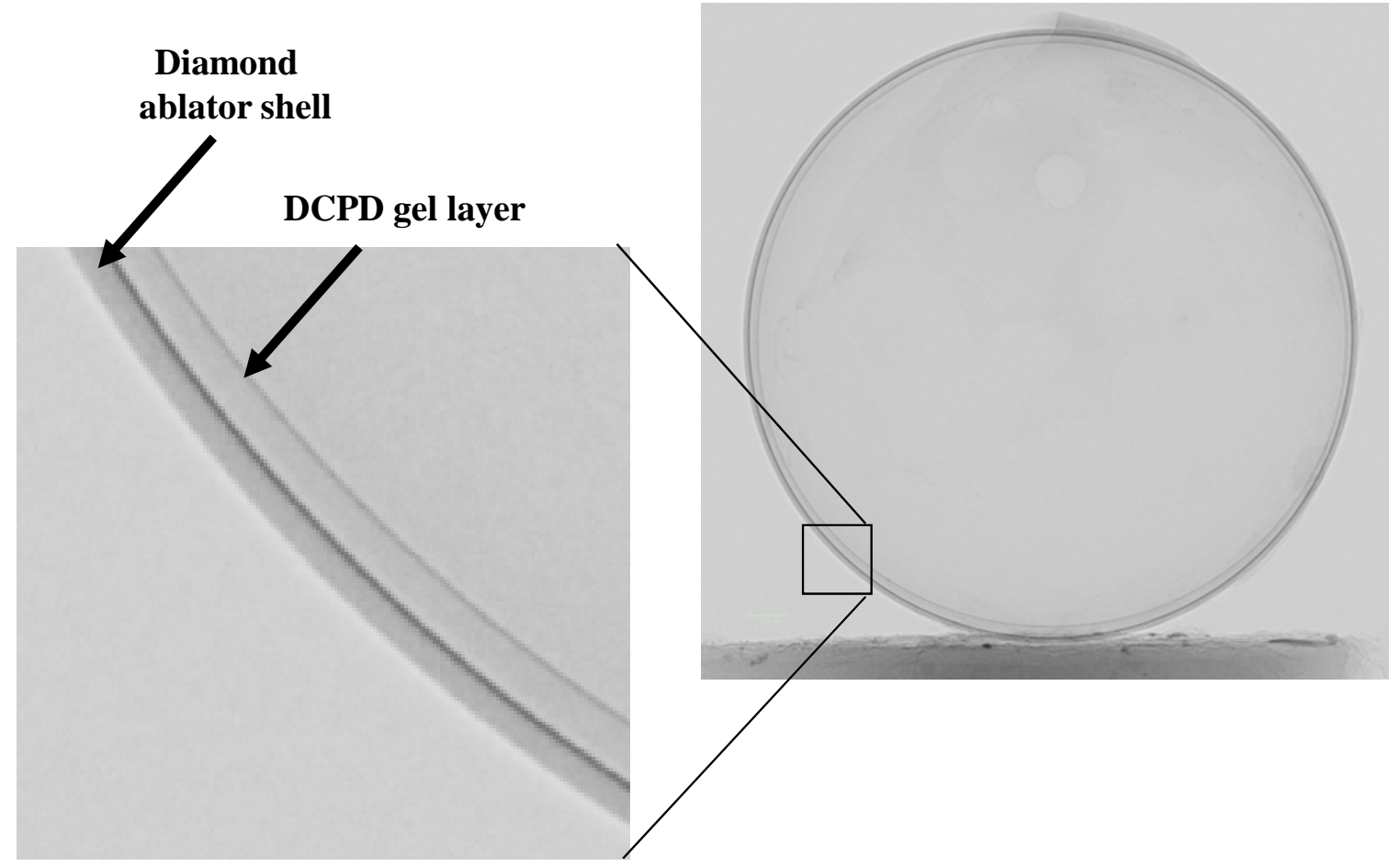

Figure 8: Radiograph of a 30 micron thick DCDP gel film (30 mg/cc) formed inside a diamond ablator capsule

We therefore decided to design a random position machine (RPM) ${ }^{22}$ that provides a deterministic, continuous random change in orientation relative to the gravity vector thus simulating a true microgravity environment. This can be achieved by a system that uses two perpendicular and independently driven frames. The machine is now being tested, and preliminary results using silica aerogels look very promising. 


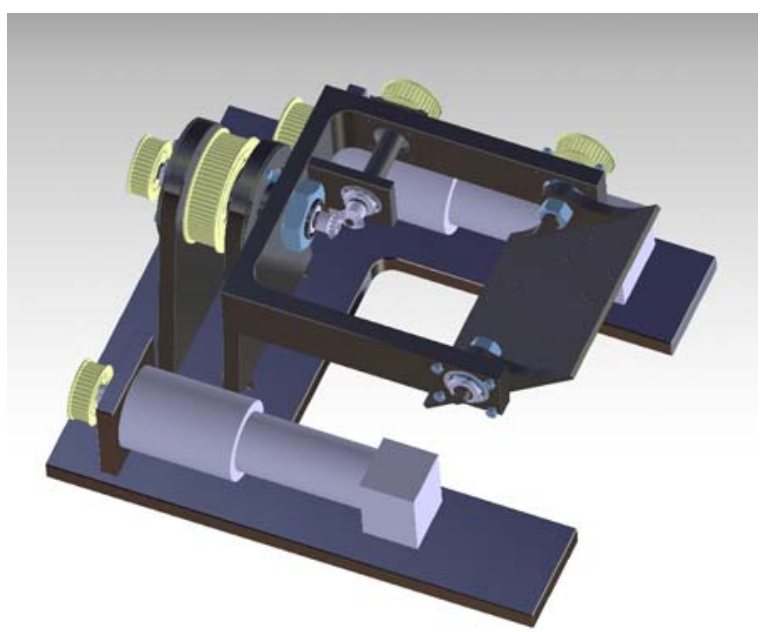

Figure 9: Computer model of the random positioning machine with two independently driven perpendicular frames. The sphere filled with the foam precursor solution is mounted on the inner stage, in the center of rotation.

\subsection{Doping of the foam inside the ablator capsule}

The purpose of the thin foam shell inside fusion application targets is the requirement to bring high-Z dopants in close contact with the DT fuel. There are two options to fabricate doped foam shells: 1) Adding modified monomers to the polymer solution, and 2) doping of the final aerogel structure. We decided to explore the latter route by using the reactivity of the remaining $\mathrm{C}=\mathrm{C}$ double bonds in the DCPD polymer to attach volatile high-Z species such as iodine. The technique is experimental very simple, and consists of exposing the foam-filled ablator shell to volatile reactants such as iodine. The radiograph of an iodine-doped foam inside a diamond shell is shown in Figure 10.
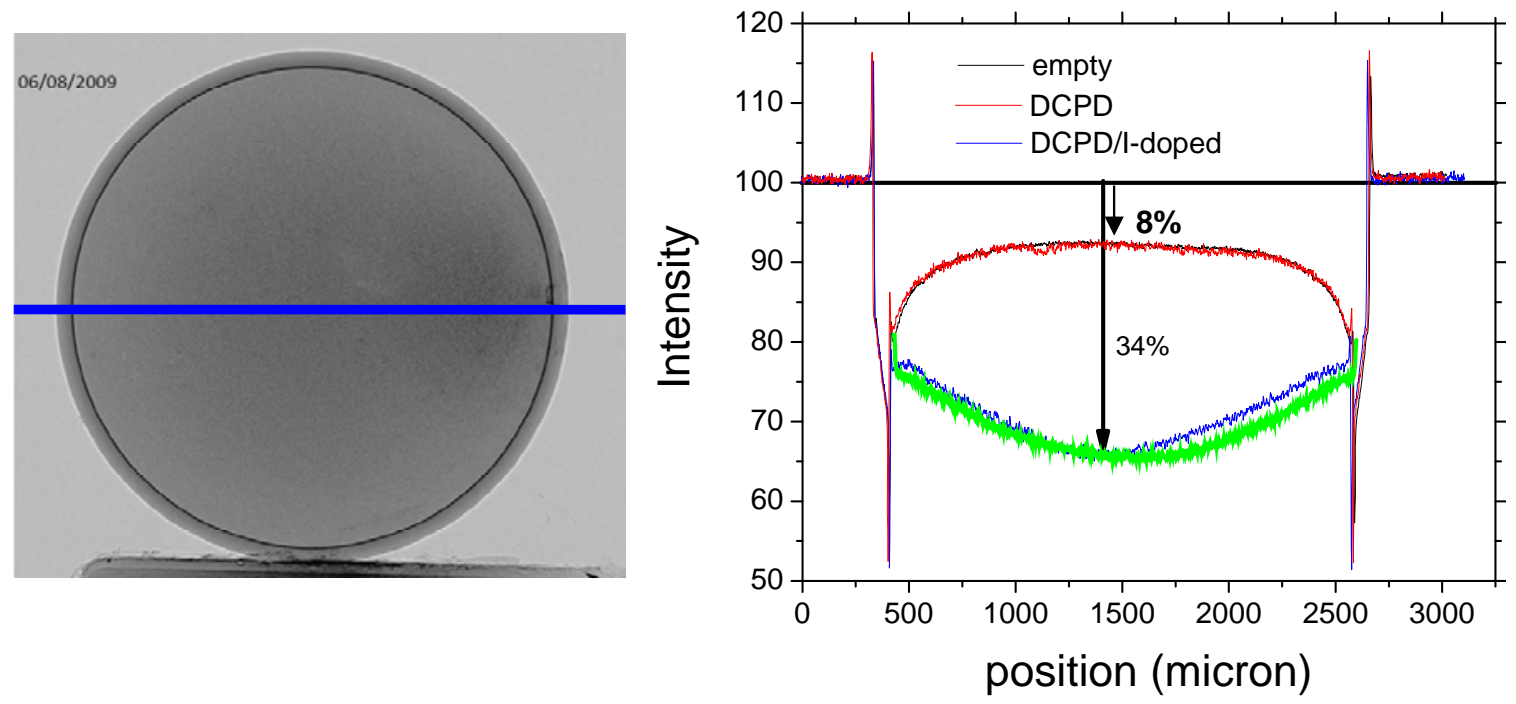

Figure 10: (left) Radiograph of an iodine-doped completely-filled foam target. (right) Line intensity profile of the empty diamond shell (black) as well as of the foam filled shell before (red) and after (blue) iodine doping. Note that the undoped foam exhibits too little x-ray contrast to be detected. The green curve is the predicted intensity profile from a doped foam sphere. 


\section{Summmary}

In summary, we have demonstrated the feasibility of all process steps required to fabricate foam-lined uses-of-ignition targets based on "chemistry in-a-capsule approach": We have systematically studied the formation of thin films in a rotating cylinder geometry, and applied the results to demonstrate the formation of thin gel layers on the inside of a spherical ablator shell. We have developed a non-shrinking $30 \mathrm{mg} / \mathrm{cc}$ hydrocarbon polymer airogel and studied its rheological properties during gel formation. We also studied the wetting behavior of various low-density aerogels with cryogenic hydrogen using both free-standing aerogel samples as well as aerogels formed inside a spherical ablator shell. Our results demonstrate that even a $30 \mathrm{mg} / \mathrm{cc}$ hydrocarbon polymer aerogel can be strong enough to survive wetting with cryogenic hydrogen. Finally, we have incorporated high-Z dopants such as iodined into free-standing aerogel samples as well as into aerogels inside a spherical ablator shell.

This work performed under the auspices of the U. S. Department of Energy by Lawrence Livermore National Laboratory under Contract DE-AC52-07NA27344. 


\section{References:}

1. R. R. Paguio, C. A. Frederick, J. F. Hund, D. G. Czechowicz, A. Nikroo, M. Takagi, O. Acenas, M. Thi and Ieee, in 21st IEEE/NPSS Symposium on Fusion Engineering - SOFE 05, 2006, pp. 85-89.

2. R. R. Paguio, M. Takagi, M. Thi, J. F. Hund, A. Nikroo, S. Paguio, R. Luo, A. L. Greenwood, O. Acenas and S. Chowdhury, Improving the wall uniformity of resorcinol formaldehyde foam shells by modifying emulsion components, San Diego, CA, 2006.

3. M. Takagi, M. Ishihara, T. Norimatsu, T. Yamanaka, Y. Izawa and S. Nakai, J. Vac. Sci. Technol. A, 1993, 11, 2837-2845.

4. M. Takagi, T. Norimatsu, T. Yamanaka and S. Nakai, J. Vac. Sci. Technol. A, 1991, 9, 2145-2148.

5. A. Nikroo, D. Czechowicz, R. Paguio, A. L. Greenwood and M. Takagi, Fusion Sci. Technol., 2004, 45, 84-89.

6. G. E. Overturf, R. Cook, S. A. Letts, S. R. Buckley, M. R. McClellan and D. SchroenCarey, Fusion Technology, 1995, 28, 1803-1808.

7. D. Schroen-Carey, G. E. Overturf, R. Reibold, S. R. Buckley, S. A. Letts and R. Cook, J. Vac. Sci. Technol. A, 1995, 13, 2564-2568.

8. F. Ito, K. Nagai, M. Nakai, T. Norimatsu, A. Nikitenko, S. Tolokonnikov, E. Koresheva, T. Fujimura, H. Azechi and K. Mima, Japanese Journal of Applied Physics Part 2-Letters \& Express Letters, 2006, 45, L335-L338.

9. $\quad$ K. Nagai, H. Yang, T. Norimatsu, H. Azechi, F. Belkada, Y. Fujimoto, T. Fujimura, K. Fujioka, S. Fujioka, H. Homma, F. Ito, A. Iwamoto, T. Jitsuno, Y. Kaneyasu, M. Nakai, N. Nemoto, H. Saika, T. Shimoyama, Y. Suzuki, K. Yamanaka and K. Mima, Nucl. Fusion, 2009, 49.

10. H. Yang, K. Nagai, N. Nakai and T. Norimatsu, Laser Part. Beams, 2008, 26, 449-453.

11. R. A. Sacks and D. H. Darling, Nucl. Fusion, 1987, 27, 447-452.

12. S. M. Lambert, G. E. Overturf, G. Wilemski, S. A. Letts, D. SchroenCarey and R. C. Cook, Journal of Applied Polymer Science, 1997, 65, 2111-2122.

13. R. W. Pekala, J. Mater. Sci., 1989, 24, 3221-3227.

14. C. A. Frederick, R. R. Paguio, A. Nikroo, J. H. Hund, O. Acennas and M. Thi, Fusion Sci. Technol., 2006, 49, 657-662.

15. S. A. Letts, S. R. Buckley, F.-M. Kong, E. F. Lindsay and M. L. Sattler, Mat. Res. Soc. Symp. Proc., 1989, 177, 275-279.

16. J. Streit and D. Schroen, Fusion Sci. Technol., 2003, 43, 321-326.

17. K. Nagai, T. Norimatsu and Y. Izawa, Fusion Sci. Technol., 2004, 45, 79-83.

18. M. R. Kessler and S. R. White, Journal of Polymer Science Part A: Polymer Chemistry, 2002, 40, 2373-2383.

19. P. Schwab, R. H. Grubbs and J. W. Ziller, J. Am. Chem. Soc., 1996, 118, 100-110.

20. M. Kessler, G. Larin and N. Bernklau, Journal of Thermal Analysis and Calorimetry, 2006, 85, 7-12.

21. A. G. M. Barrett, B. T. Hopkins and J. Kobberling, Chemical Reviews, 2002, 102, 3301-3323. 
22. A. Borst and J. van Loon, Microgravity Science and Technology, 2009, 21, 287292. 